\title{
Controlling Excited-State Contributions to Nucleon Isovector Charges using Distillation
}

\section{Colin Egerer*}

Department of Physics, William and Mary, Williamsburg, VA 23185, USA

E-mail: cpegerer@email.wm.edu

\section{David Richards}

Thomas Jefferson National Accelerator Facility, Newport News, VA 23606, USA

E-mail: dgr@jlab.org

\section{Frank Winter}

Thomas Jefferson National Accelerator Facility, Newport News, VA 23606, USA

E-mail: fwinter@jlab.org

\begin{abstract}
Reliable calculations in Lattice QCD must confront excited-state contamination and statistical precision, where attempts to reduce excited-state effects are often handled with various "smearing" algorithms. Distillation, a particularly powerful form of smearing, has seen extensive use in spectroscopy calculations, yet, until now, has not seen use in calculations of hadronic structure. We find that distillation, and an extended basis of distilled interpolators facilitated by the variational method, applied to calculations of the scalar, axial and tensor isovector charges of the nucleon, lead to a dramatic reduction in statistical uncertainty and excited-state contamination when compared to conventional methods, thereby making a strong case for distillation in future, more elaborate, structure calculations.
\end{abstract}

The 36th Annual International Symposium on Lattice Field Theory - LATTICE2018

22-28 July, 2018

Michigan State University, East Lansing, Michigan, USA.

\footnotetext{
* Speaker.
} 


\section{Introduction}

As all lattice calculations of nucleon properties are subject to a trade-off between excited-state contamination at short Euclidean times and a degrading signal-to-noise ratio at large Euclidean times, the need for tamed excited-state effects is paramount. Perhaps the most notoriously contaminated signal in lattice calculations is that of the axial charge of the nucleon. The flavor non-singlet axial vector current $A_{\mu}^{3}=\bar{\psi} \gamma_{\mu} \gamma_{5} \frac{\tau^{3}}{2} \psi$ between incoming and outgoing nucleons is expressible as

$$
\left\langle N_{s^{\prime}}\left(p^{\prime}\right)\left|A_{\mu}^{3}\right| N_{s}(p)\right\rangle=\bar{u}_{N}^{s^{\prime}}\left(p^{\prime}\right)\left[\gamma_{\mu} \gamma_{5} G_{A}\left(q^{2}\right)+\frac{q_{\mu}}{2 M_{N}} \gamma_{5} G_{P}\left(q^{2}\right)+i \frac{\sigma^{\mu v} q_{v}}{2 M_{N}} \gamma_{5} G_{T}\left(q^{2}\right)\right] \frac{\tau^{3}}{2} u_{N}^{s}(p)
$$

where $\left\{s^{\prime}, s\right\}$ the polarizations, $q_{\mu}=p_{\mu}^{\prime}-p_{\mu}$ the momentum transfer, and $G_{P}\left(q^{2}\right)$ and $G_{T}\left(q^{2}\right)$ the induced pseudoscalar and tensor form factors. The forward limit, with $q_{\mu}=0$, defines the axial charge $g_{A}^{u-d}=G_{A}(0)$; this simple definition has elevated its calculation to a benchmark quantity. Recent work motivated by the Feynman-Hellman theorem [1] and high-statistics studies at large source-sink separations [2,3] have reconciled longstanding tension between the experimental and lattice determinations of $g_{A}^{u-d}$. Nevertheless, it is curious that the bulk of lattice calculations routinely underdetermine the nucleon axial charge by $\sim 10-15 \%$. The reader is referred to $[4,5,6,7,8,9,10]$ for recent calculations.

Systematic uncertainties in calculations of nucleon charges are predominantly due to the inability of a lattice interpolating field to isolate the ground-state nucleon from its excitations and multi-particle states of the same lattice quantum numbers. Spatial smearing and the variational method are widely used techniques to overcome this hurdle. An alternative smearing algorithm called "Distillation" [11] has garnered much attention in spectroscopy calculations for its utility in efficiently identifying a plethora of hadronic states across a wide range of quantum numbers, including some of hybrid nature. Motivated by this success, we explore the use of distillation in the calculation of nucleon isovector charges $g_{A}^{u-d}$ and $g_{T}^{u-d}$. A complete accounting of $g_{S}^{u-d}, g_{A}^{u-d}$ and $g_{T}^{u-d}$ can be found in [12].

We show distillation provides a significant reduction in statistical uncertainty and appears to have a better control over excited-state effects in nucleon matrix elements, when compared to local interpolators smeared in a conventional manner. The taming of excited-states aligns with the construction of distillation as a low-mode projection of conventional smearing kernels, while the improved systematics follows from distillation enabling explicit momentum projections at all time slices in a correlation function. Construction of a suitable basis of distilled interpolators, together with the variational method, illustrates further improvements in nucleon matrix elements over standard smearing methods. A study of the spectra of the excited baryon states on this same ensemble is presented elsewhere [13].

\section{Smearing and Distillation}

Spectral decompositions of momentum-projected two-point $C_{2 \text { pt }}(t)=\sum_{\vec{x}} e^{-i \vec{p} \cdot \vec{x}}\langle\mathscr{O}(\vec{x}, t) \overline{\mathscr{O}}(0)\rangle$ and three-point $C_{3 \mathrm{pt}}(t, \tau)=\sum_{\vec{x}, \vec{y}} e^{-i \overrightarrow{p^{\prime}} \cdot \vec{x}} e^{i \vec{q} \cdot \vec{y}}\langle\mathscr{O}(\vec{x}, t) \mathscr{J}(\vec{y}, \tau) \overline{\mathscr{O}}(0)\rangle$ functions demonstrate groundstate hadronic properties are identified in the large-time regime (i.e. $0 \ll \tau \ll t$ ) or with a judicial 
choice for $\mathscr{O}$. Spatial smearing, such as Jacobi smearing, improves overlap of $\mathscr{O}$ onto low-lying states via successive application of the kernel $\left(1+\sigma \nabla^{2}(t) / n_{\sigma}\right)^{n_{\sigma}}$ to point-like quark/gluon fields.

Distillation is defined as a low-rank approximation to some gauge-covariant smearing kernel often the Jacobi-smearing kernel. By seeking solutions to $-\nabla^{2}(t) \xi^{(k)}(t)=\lambda^{(k)}(t) \xi^{(k)}(t)$, ordering solutions according to the magnitude of the eigenvalues, the distillation operator is constructed as the outer-product of two eigenvectors on some time slice

$$
\square(\vec{x}, \vec{y} ; t)_{a b}=\sum_{k=1}^{N} \xi_{a}^{(k)}(\vec{x}, t) \xi_{b}^{(k) \dagger}(\vec{y}, t),
$$

with color indices $\{a, b\}$. The components of a correlation function are constructed from

$$
\begin{gathered}
\text { Solution vectors: } \quad S_{\alpha \beta}^{(k)}\left(\vec{x}, t^{\prime} ; t\right)=M_{\alpha \beta}^{-1}\left(t^{\prime}, t\right) \xi^{(k)}(t) \\
\text { Perambulators: } \quad \tau_{\alpha \beta}^{k l}\left(t^{\prime}, t\right)=\xi^{(k) \dagger}\left(t^{\prime}\right) M_{\alpha \beta}^{-1}\left(t^{\prime}, t\right) \xi^{(l)}(t) \\
\text { Elementals: } \quad \Phi_{\mu \nu \rho}^{(i, j, k)}(t)=\varepsilon^{a b c}\left(D_{1} \xi^{(i)}\right)^{a}\left(D_{2} \xi^{(j)}\right)^{b}\left(D_{3} \xi^{(k)}\right)^{c}(t) S_{\mu v \rho}
\end{gathered}
$$

where $S_{\mu v \rho}$ are subduction matrices, $M$ the Dirac operator, and $D_{n}$ a covariant derivative acting on the $n^{\text {th }}$ quark of the nucleon interpolator. We note here introduction of gauge-covariant derivatives probes the radial/angular structure of the nucleon. Distillation factorizes quark propagation from interpolator construction, allowing arbitrarily complicated interpolators to be correlated once a single set of perambulators have been computed. Moreover, distillation ensures a greater sampling of configurations via explicit momentum projection at source, sink, and insertion.

\section{Lattice Parameters}

Calculations were performed on a $32^{3} \times 64$ lattice ensemble with an inverse coupling $\beta=6.3$, and $2+1$ quark flavors described with the clover-Wilson fermion action. The needed two- and threepoint correlation functions were calculated on 350 configurations, separated by 10 updates in the HMC algorithm. This lattice ensemble was found to have a lattice spacing of $a=0.09840(4) \mathrm{fm}$ via the Wilson-flow scale $w_{0}$, yielding $m_{\pi}=356 \mathrm{MeV}$ and $m_{\pi} L \simeq 5.7$.

In this work we study zero-momentum nucleons, polarized along the z-axis, interpolated from the vacuum with four different types of operators - three distilled (two of which are supplemented with the variational method) and one Jacobi-smeared interpolator. The simplest nucleon interpolator consistent with the quantum numbers of the nucleon is

$$
\mathscr{N}(x)=\varepsilon^{a b c}\left[u^{a \top}(x) C \frac{\left(1 \pm \gamma_{4}\right)}{2} \gamma_{5} d^{b}(x)\right] u_{\alpha}^{c}(x)
$$

where $C=\gamma_{2} \gamma_{4}$ the charge conjugation matrix, $\{a, b, c\}$ color indices, and $\alpha$ a free Dirac index. The signal-to-noise ratio of the forward (backward) propagating states is improved following application of the non-relativistic projector $\left(1 \pm \gamma_{4}\right) / 2$. Sixty applications of the Jacobi-smearing kernel $(\sigma=5.0)$ to $\mathscr{N}$ defines our Jacobi-smeared interpolator, which we refer to herein as "Jacobi-SS". 
Projectors $\mathscr{P}^{2 \mathrm{pt}}=\left(1+\gamma_{4}\right) / 2$ and $\mathscr{P}^{3 \mathrm{pt}}=\mathscr{P}^{2 \mathrm{pt}}\left(1+i \gamma_{5} \gamma_{3}\right)$ are needed to project onto the forwardpropagating positive-parity, $z$-polarized nucleon:

$$
C^{2 \mathrm{pt}}(t)=\sum_{\vec{x}}\left\langle\mathscr{P}_{\beta \alpha}^{2 \mathrm{pt}} \mathscr{N}_{\alpha}(\vec{x}, t) \overline{\mathscr{N}}_{\beta}(0)\right\rangle \quad \text { and } \quad C^{3 \mathrm{pt}}(t, \tau)=\sum_{\vec{x}, \vec{z}}\left\langle\mathscr{P}_{\beta \alpha}^{3 \mathrm{pt}} \mathscr{N}_{\alpha}(\vec{x}, t) \mathscr{O}_{\Gamma}^{u-d}(\vec{z}, \tau) \overline{\mathscr{N}}_{\beta}(0)\right\rangle
$$

where $\mathscr{O}_{\Gamma}^{u-d}$ is the isovector insertion. The sequential-source method is used in three-point calculations employing $\mathscr{N}$, reducing distinct inversions of the Dirac operator.

We employ a distillation space of rank 64, with 10 iterations of stout smearing $\left(\rho_{i j}=0.08\right.$ and $\rho_{\mu 4}=\rho_{4 \mu}=0$ ) applied to the links on each time slice. The first distilled interpolator we consider is referred to as the ${ }^{2} S_{S} \frac{1}{2}^{+}$, the closest non-relativistic analogue of 3.1. Application of the variational method to two different bases of distilled interpolators defines our final two interpolators:

$$
\begin{aligned}
& \mathscr{B}_{3}=\left\{{ }^{2} S_{S} \frac{1}{2}^{+},{ }^{4} P_{M} \frac{1}{2}^{+},{ }^{4} D_{M} \frac{1}{2}^{+}\right\} \quad \longrightarrow \quad \hat{\mathscr{P}}_{3} \\
& \mathscr{B}_{7}=\left\{{ }^{2} S_{S} \frac{1^{+}}{}{ }^{2},{ }^{2} S_{M} \frac{1}{2}^{+},{ }^{2} S_{S}^{\prime} \frac{1}{2}^{+},{ }^{2} P_{A} \frac{1}{2}^{+},{ }^{2} P_{M} \frac{1}{2}^{+},{ }^{4} P_{M} \frac{1}{2}^{+},{ }^{4} D_{M} \frac{1}{2}^{+}\right\} \quad \longrightarrow \quad \hat{\mathscr{P}}_{7} .
\end{aligned}
$$

The reader is referred to [12] for explicit construction of these interpolators. Due to the high Wick contraction costs of distillation, we applied the variational method to a correlation matrix of two-point functions, fixing $\hat{\mathscr{P}}_{3 / 7}$ for use in the three-point calculations. We note that ${ }^{4} P_{M} \frac{1}{2}^{+}$and ${ }^{4} D_{M} \frac{1}{2}^{+}$are explicitly of hybrid character - inspired by [14] where ${ }^{2} S_{S} \frac{1}{2}^{+}$and these hybrid operators were found to have optimal overlap onto the ground-state nucleon.

Spectral decompositions of the projected correlation functions of Eqn. 3.2 show the correlators behave as $C^{2 \mathrm{pt}}(t)=2 \sum_{n}\left|Z_{n}\right|^{2} e^{-M_{n} t}$ and $C^{3 \mathrm{pt}}\left(t_{\text {sep }}, \tau\right)=\left(\frac{\left|Z_{0}\right|^{2}}{4 M_{0}^{2}} \mathscr{J}_{00} e^{-M_{0} t_{\text {sep }}}+\frac{\left|Z_{1}\right|^{2}}{4 M_{1}^{2}} \mathscr{J}_{11} e^{-M_{1} t_{\text {sep }}}\right)+$ $\frac{Z_{0} Z_{1}}{2 M_{0} M_{1}} \mathscr{J}_{01} e^{-\frac{\left(M_{1}+M_{0}\right)}{2} t_{\text {sep }}} \cosh \left[\left(M_{1}-M_{0}\right)\left(\tau-\frac{t_{\text {sep }}}{2}\right)\right]$. Use of distilled interpolators introduces an overall factor of the lattice volume $V_{3}$, due to a spatial sum at the source. We therefore perform simultaneous fits to our two-/three-point correlators

$$
\begin{gathered}
C_{\text {fit }}^{2 \mathrm{pt}}(t)=e^{-M_{0} t}\left[|\mathbf{a}|^{2}+|\mathbf{b}|^{2} e^{-\left(M_{1}-M_{0}\right) t}\right] \\
C_{\text {fit }}^{3 \mathrm{pt}}\left(t_{\mathrm{sep}}, \tau\right)=e^{-M_{0} t_{\mathrm{sep}}}\left(\mathscr{A}+\mathscr{B} e^{-\Delta m t_{\mathrm{sep}}}+\mathscr{C} e^{-\Delta m \frac{t_{\mathrm{sep}}}{2}} \cosh \left[\left(M_{1}-M_{0}\right)\left(\tau-\frac{t_{\mathrm{sep}}}{2}\right)\right]\right)
\end{gathered}
$$

to quantify the ground and first-excited state masses, overlap factors, and the desired ground-state matrix element $g_{00}^{\Gamma}=\mathscr{A} /|\mathbf{a}|^{2}$, while accounting for all correlations between our data.

\section{Results \& Discussion}

Given the use of a single ensemble, we present only unrenormalized charges to demonstrate the improvements afforded by distillation. We compute the two(three)-point correlation functions averaged over three(one) source positions. The three-point functions are calculated for $t_{\text {sep }} \in$ $\{8,12,16\}$, with currents inserted for $\tau \in\left[0, t_{\text {sep }}-1\right]$. We first judge the "quality" of an interpolator by plotting, as a function of the source-sink separation $t$, the effective mass $M_{\text {eff }}(t+0.5)=$ $(1 / a) \ln \left[C^{2 \mathrm{pt}}(t) / C^{2 \mathrm{pt}}(t+1)\right]$. 
It is clear from Fig. 1, the use of distillation affords plateaus in the nucleon effective mass that begin for source-sink separations $t \sim 0.6 \mathrm{fm}$ and demonstrate roughly a factor of 2 improvement in statistics - most notable is the reduction in error for large Euclidean times. The application of the variational method, as expected, leads to a greater exponential decay of excited-states at early Euclidean times, and an effective mass plateau that begins one time slice earlier in the case of $\hat{\mathscr{P}}_{7}$. We attribute the increase in latetime noise in $\hat{\mathscr{P}}_{3}$ and $\hat{\mathscr{P}}_{7}$, compared to ${ }^{2} S_{2} \frac{1}{2}^{+}$, to result from the elements of the correlation matrix being dominated by noise at large sourcesink separations. For brevity we present results for the isovector charges where $t_{2 \mathrm{pt}}^{\mathrm{fit}} \in[2,16]$ and $\tau_{\mathrm{fit}} \in\left[2, t_{\mathrm{sep}}-2\right]$. The reader is referred to [12] for a complete analysis of the dependence of our results on the fitting windows chosen, as well as results for the scalar and vector charges, and a complete listing of our fitted parameters. We define an effective charge $g_{\Gamma}^{\text {eff }}(t, \tau)=C_{\Gamma}^{3 \mathrm{pt}}(t, \tau) / C_{\mathrm{fit}}^{2 \mathrm{pt}}(t)$ to quantify the amount of excited-state contamination, where $C_{\mathrm{fit}}^{2 \mathrm{pt}}(t)$ is the best fit applied to the two-point function and $C_{\Gamma}^{3 \mathrm{pt}}(t, \tau)$ the three-point function of the same source-sink separation and interpolator constructions. The errors (gray) of our extracted isovector charges (black lines) are estimated via a simultaneous jackknife resampling of the three-point correlator and two-point fit.

In figures 2 and 3 we display our calculated effective matrix elements, simultaneous fits, and extracted isovector charges. Distillation appears to offer the combined benefit of improved statistical precision and controlled excited-state effects in nucleon matrix elements, relative to Jacobi smeared interpolators. Most notable are the broad, consistent plateaus that are present in the effective matrix elements calculated using distillation. Although the gains of the variationally-improved distilled interpolators compared to the local distilled interpolator are less dramatic, the results are no less significant as the effective matrix elements were found to be more consistent for different $t_{\text {sep }}$ and required no additional Dirac inversions to do so. Having demonstrated the utility of distillation in structure calculations, the next step in this program is to repeat this work for moving states
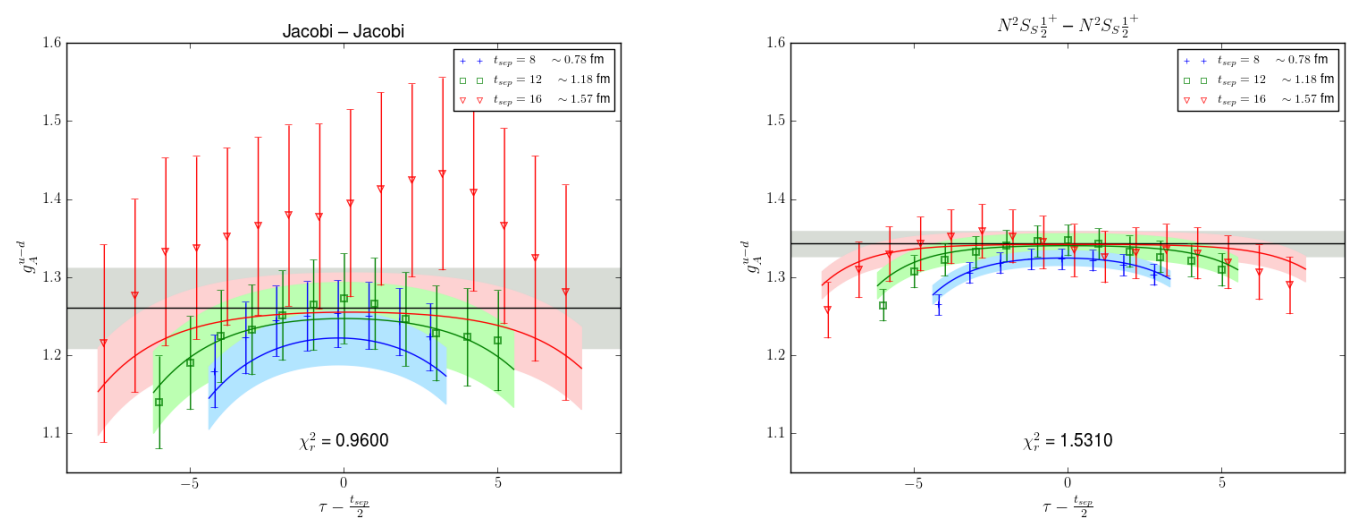

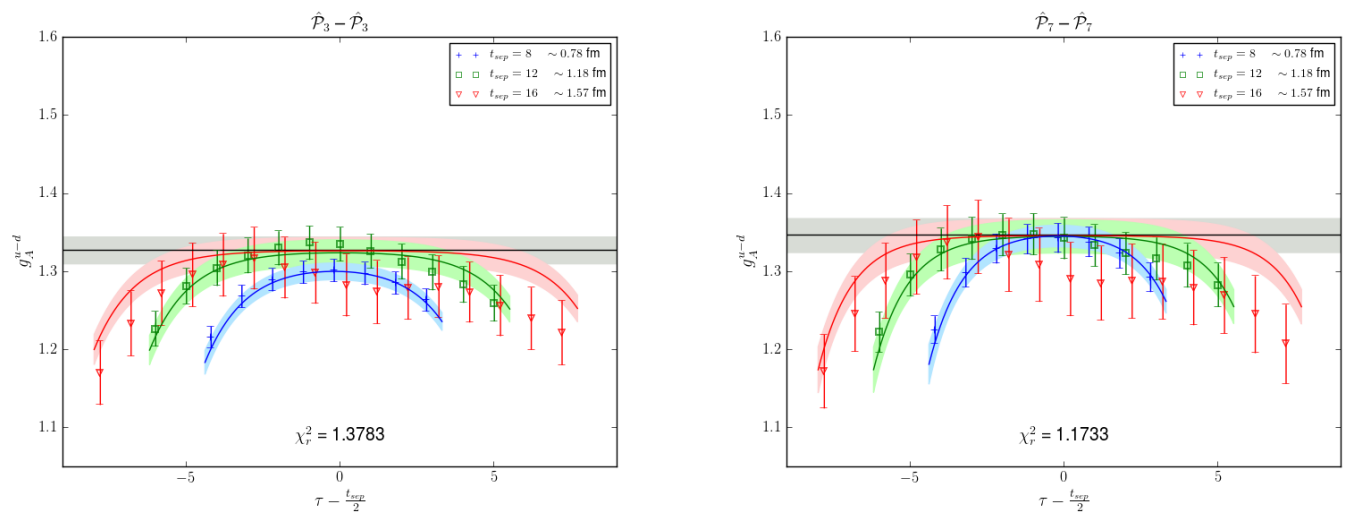

Figure 2: Extracted and effective unrenormalized isovector axial charge using the Jacobi-SS (upper-left), ${ }^{2} S_{S} \frac{1}{2}^{+}$(upper-right), $\hat{\mathscr{P}}_{3}$ (lower-left) and the $\hat{\mathscr{P}}_{7}$ (lower-right).
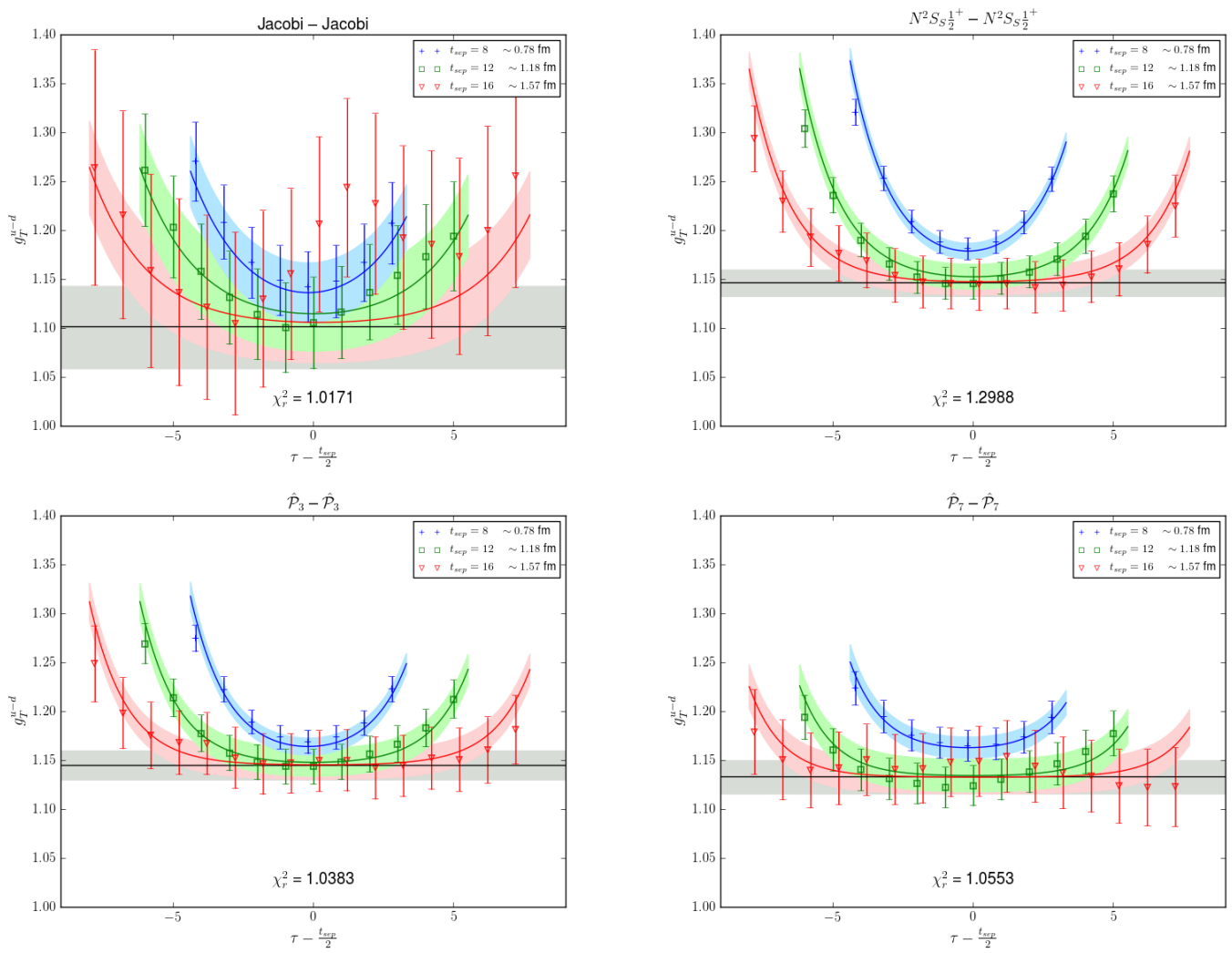

Figure 3: Extracted and effective unrenormalized isovector tensor charge using the Jacobi-SS (upper-left), ${ }^{2} S_{S} \frac{1}{2}^{+}$(upper-right), $\hat{\mathscr{P}}_{3}$ (lower-left) and the $\hat{\mathscr{P}}_{7}$ (lower-right).

and generalize to off-forward scattering.

\section{Acknowledgments}

Computations for this work were carried out in part on facilities of the USQCD Collaboration, 
which are funded by the Office of Science of the U.S. Department of Energy, using the Chroma [15] software suite. This material is based upon work supported by the U.S. Department of Energy, Office of Science, Office of Nuclear Physics under contracts DE-AC05-06OR23177 and DEFG02-04ER41302. Computing resources at the National Energy Research Scientific Computing Center (DE-AC02-05CH11231) and the Oak Ridge Leadership Computing Facility (DE-AC0500OR22725), which are DOE Office of Science User facilities, were also used. CE was supported in part by a DOE Office of Science Graduate Student Research (SCGSR) fellowship.

\section{References}

[1] C. C. Chang et al., A per-cent-level determination of the nucleon axial coupling from quantum chromodynamics, Nature 558 (2018) 91 [1805.12130].

[2] G. S. Bali, S. Collins, B. Glässle, M. Göckeler, J. Najjar, R. H. Rödl et al., Nucleon isovector couplings from $N_{f}=2$ lattice QCD, Phys. Rev. D91 (2015) 054501 [1412.7336].

[3] R. Horsley, Y. Nakamura, A. Nobile, P. E. L. Rakow, G. Schierholz and J. M. Zanotti, Nucleon axial charge and pion decay constant from two-flavor lattice QCD, Phys. Lett. B732 (2014) 41 [1302.2233].

[4] H.-W. Lin, R. Gupta, B. Yoon, Y.-C. Jang and T. Bhattacharya, Quark contribution to the proton spin from 2+1+1-flavor lattice QCD, 1806.10604 .

[5] RBC, UKQCD, LHP collaboration, S. Ohta, Some nucleon isovector obsesrvables from 2+1-flavor domain-wall QCD at physical mass, PoS LATTICE2015 (2016) 124 [1511.05126].

[6] J. Liang, Y.-B. Yang, K.-F. Liu, A. Alexandru, T. Draper and R. S. Sufian, Lattice Calculation of Nucleon Isovector Axial Charge with Improved Currents, Phys. Rev. D96 (2017) 034519 [1612.04388].

[7] R. Gupta, Y.-C. Jang, B. Yoon, H.-W. Lin, V. Cirigliano and T. Bhattacharya, Isovector Charges of the Nucleon from 2+1+1-flavor Lattice QCD, Phys. Rev. D98 (2018) 034503 [1806. 0900 6].

[8] C. Alexandrou, M. Constantinou, K. Hadjiyiannakou, K. Jansen, C. Kallidonis, G. Koutsou et al., Nucleon axial form factors using $N_{f}=2$ twisted mass fermions with a physical value of the pion mass, Phys. Rev. D96 (2017) 054507 [1705.03399].

[9] B. Yoon et al., Isovector charges of the nucleon from 2+1-flavor QCD with clover fermions, Phys. Rev. D95 (2017) 074508 [1611.07452].

[10] B. J. Owen, J. Dragos, W. Kamleh, D. B. Leinweber, M. S. Mahbub, B. J. Menadue et al., Variational Approach to the Calculation of gA, Phys. Lett. B723 (2013) 217 [1212 . 4668].

[11] Hadron Spectrum collaboration, M. Peardon, J. Bulava, J. Foley, C. Morningstar, J. Dudek, R. G. Edwards et al., A Novel quark-field creation operator construction for hadronic physics in lattice QCD, Phys. Rev. D80 (2009) 054506 [0 905 .2160].

[12] C. Egerer, D. Richards and F. Winter, Controlling Excited-State Contributions with Distillation in Lattice QCD Calculations of Nucleon Isovector Charges $g_{S}^{u-d}, g_{A}^{u-d}, g_{T}^{u-d}, 1810.09991$.

[13] T. Khan et al., "Positive-parity Baryon Spectra on Isotropic Lattice." These proceedings, 2018.

[14] J. J. Dudek and R. G. Edwards, Hybrid Baryons in QCD, Phys. Rev. D85 (2012) 054016 [1201.2349].

[15] SCIDAC, LHPC, UKQCD collaboration, R. G. Edwards and B. Joo, The Chroma software system for lattice QCD, Nucl. Phys. Proc. Suppl. 140 (2005) 832 [hep-lat/ 0409003 ]. 